\title{
Phenome-wide association analysis of LDL- cholesterol lowering genetic variants in PCSK9
}

Amand F. Schmidt ${ }^{1,2,3^{*+}}$ DD, Michael V. Holmes ${ }^{4+}$, David Preiss ${ }^{4 \dagger}$, Daniel I. Swerdlow ${ }^{1,5}$, Spiros Denaxas ${ }^{3,6,7,8}$, Ghazaleh Fatemifar ${ }^{3,6,7}$, Rupert Faraway ${ }^{1}$, Chris Finan ${ }^{1,3}$, Dennis Valentine ${ }^{3,9}$, Zammy Fairhurst-Hunter ${ }^{10}$, Fernando Pires Hartwig ${ }^{11}$, Bernardo Lessa Horta ${ }^{11}$, Elina Hypponen ${ }^{12,13,14}$, Christine Power ${ }^{13}$, Max Moldovan ${ }^{14}$, Erik van Iperen ${ }^{15,16}$, Kees Hovingh ${ }^{17}$, Ilja Demuth ${ }^{18,19}$, Kristina Norman ${ }^{20,21,22}$, Elisabeth Steinhagen-Thiessen ${ }^{18}$, Juri Demuth ${ }^{23}$, Lars Bertram², ${ }^{24,25}$, Christina M. Lill ${ }^{26,27,28}$, Stefan Coassin ${ }^{29}$, Johann Willeit ${ }^{30}$, Stefan Kiechl ${ }^{30}$, Karin Willeit ${ }^{30,31}$, Dan Mason ${ }^{32}$, John Wright ${ }^{32}$, Richard Morris ${ }^{33}$, Goya Wanamethee ${ }^{33}$, Peter Whincup ${ }^{34}$, Yoav Ben-Shlomo ${ }^{35}$, Stela McLachlan ${ }^{36}$, Jackie F. Price ${ }^{36}$, Mika Kivimaki ${ }^{37}$, Catherine Welch ${ }^{37}$, Adelaida Sanchez-Galvez ${ }^{37}$, Pedro Marques-Vidal ${ }^{38}$, Andrew Nicolaides ${ }^{39,40}$, Andrie G. Panayiotou ${ }^{41}$, N. Charlotte Onland-Moret ${ }^{42}$, Yvonne T. van der Schouw ${ }^{42}$, Giuseppe Matullo ${ }^{43,44}$, Giovanni Fiorito ${ }^{43,44}$, Simonetta Guarrera ${ }^{43,44}$, Carlotta Sacerdote ${ }^{45}$, Nicholas J. Wareham ${ }^{46}$, Claudia Langenberg ${ }^{46}$, Robert A. Scott ${ }^{46}$, Jian'an Luan ${ }^{46}$, Martin Bobak ${ }^{37}$, Sofia Malyutina ${ }^{47,48}$, Andrzej Pająk ${ }^{49}$, Ruzena Kubinova ${ }^{50}$, Abdonas Tamosiunas ${ }^{51}$, Hynek Pikhart ${ }^{37}$, Niels Grarup ${ }^{52}$, Oluf Pedersen ${ }^{52}$, Torben Hansen ${ }^{52}$, Allan Linneberg ${ }^{53,54}$, Tine Jess ${ }^{54}$, Jackie Cooper ${ }^{55}$, Steve E. Humphries ${ }^{55}$, Murray Brilliant ${ }^{56}$, Terrie Kitchner ${ }^{56}$, Hakon Hakonarson ${ }^{57}$, David S. Carrell ${ }^{60}$, Catherine A. McCarty ${ }^{58}$, Kirchner H. Lester ${ }^{59}$, Eric B. Larson ${ }^{60}$, David R. Crosslin ${ }^{61}$, Mariza de Andrade ${ }^{62}$, Dan M. Roden ${ }^{63}$, Joshua C. Denny ${ }^{64}$, Cara Carty ${ }^{65}$, Stephen Hancock ${ }^{66}$, John Attia ${ }^{66,67}$, Elizabeth Holliday ${ }^{66,67}$ Rodney Scott ${ }^{66}$, Peter Schofield ${ }^{68}$, Martin O'Donnell ${ }^{69}$, Salim Yusuf ${ }^{69}$, Michael Chong ${ }^{69}$, Guillaume Pare ${ }^{69}$, Pim van der Harst ${ }^{15,16,70,71}$, M. Abdullah Said ${ }^{71}$, Ruben N. Eppinga ${ }^{71}$, Niek Verweij ${ }^{71}$, Harold Snieder ${ }^{72}$, Lifelines Cohort authors, Tim Christen ${ }^{73}$, D. O. Mook-Kanamori ${ }^{73}$, the ICBP Consortium, Stefan Gustafsson ${ }^{74}$, Lars Lind ${ }^{75,76}$, Erik Ingelsson ${ }^{74,75}$, Raha Pazoki ${ }^{77,78}$, Oscar Franco ${ }^{77}$, Albert Hofman ${ }^{77}$, Andre Uitterlinden ${ }^{77}$, Abbas Dehghan ${ }^{77}$, Alexander Teumer ${ }^{79,80}$, Sebastian Baumeister ${ }^{79,81}$, Marcus Dörr ${ }^{80,82}$, Markus M. Lerch ${ }^{83}$, Uwe Völker ${ }^{80,84}$, Henry Völzke ${ }^{79,80}$, Joey Ward ${ }^{85}$, Jill P. Pell ${ }^{85}$, Tom Meade ${ }^{86}$, Ingrid E. Christophersen ${ }^{87}$, Anke H. Maitland-van der Zee ${ }^{88,89}$, Ekaterina V. Baranova ${ }^{90}$, Robin Young ${ }^{90}$, lan Ford ${ }^{90}$, Archie Campbell ${ }^{91}$, Sandosh Padmanabhan ${ }^{92}$, Michiel L. Bots ${ }^{41}$, Diederick E. Grobbee ${ }^{41}$, Philippe Froguel ${ }^{93,94}$, Dorothée Thuillier ${ }^{93}$, Ronan Roussel ${ }^{95,96,97}$, Amélie Bonnefond ${ }^{93}$, Bertrand Cariou ${ }^{98}$, Melissa Smart ${ }^{99}$, Yanchun Bao ${ }^{100}$, Meena Kumari ${ }^{101}$, Anubha Mahajan ${ }^{100}$, Jemma C. Hopewell ${ }^{10}$, Sudha Seshadri ${ }^{101}$, the METASTROKE Consortium of the ISGC, Caroline Dale ${ }^{9}$, Rui Providencia E. Costa ${ }^{9}$, Paul M. Ridker ${ }^{102}$, Daniel I. Chasman ${ }^{102}$, Alex P. Reiner ${ }^{103}$, Marylyn D. Ritchie ${ }^{104}$, Leslie A. Lange ${ }^{105}$, Alex J. Cornish ${ }^{106}$, Sara E. Dobbins ${ }^{106}$, Kari Hemminki ${ }^{107,108}$, Ben Kinnersley ${ }^{106}$, Marc Sanson ${ }^{109,110}$, Karim Labreche ${ }^{109,110}$, Matthias Simon ${ }^{111}$, Melissa Bondy ${ }^{112}$, Philip Law ${ }^{106}$,

\footnotetext{
* Correspondence: amand.schmidt@ucl.ac.uk

${ }^{\dagger}$ Amand F Schmidt, Michael V Holmes and David Preiss are Joint first authors

${ }^{\dagger}$ Naveed Sattar, Richard Houlston, Juan P Casas and Aroon D Hingorani are Joint last authors

${ }^{1}$ Institute of Cardiovascular Science, University College London, 222 Euston

Road, London NW1 2DA, UK

${ }^{2}$ Department of Cardiology, Division Heart \& Lungs, University Medical

Center Utrecht, Utrecht University, Utrecht, the Netherlands
}

(c) The Author(s). 2019 Open Access This article is distributed under the terms of the Creative Commons Attribution 4.0 International License (http://creativecommons.org/licenses/by/4.0/), which permits unrestricted use, distribution, and reproduction in any medium, provided you give appropriate credit to the original author(s) and the source, provide a link to the Creative Commons license, and indicate if changes were made. The Creative Commons Public Domain Dedication waiver (http://creativecommons.org/publicdomain/zero/1.0/) applies to the data made available in this article, unless otherwise stated. 
Helen Speedy ${ }^{106}$, James Allan ${ }^{113}$, Ni Li ${ }^{106}$, Molly Went ${ }^{106}$, Niels Weinhold ${ }^{114}$, Gareth Morgan ${ }^{114}$, Pieter Sonneveld ${ }^{115}$, Björn Nilsson ${ }^{116}$, Hartmut Goldschmidt ${ }^{117}$, Amit Sud ${ }^{106}$, Andreas Engert ${ }^{118}$, Markus Hansson ${ }^{119,120}$, Harry Hemingway 3,6,7,121 , Folkert W. Asselbergs ${ }^{1,2,3,122}$, Riyaz S. Patel 1,3,123, Brendan J. Keating ${ }^{124}$, Naveed Sattar ${ }^{92 \dagger}$, Richard Houlston ${ }^{106+}$, Juan P. Casas ${ }^{125+}$ and Aroon D. Hingorani ${ }^{1,3+}$

\section{Abstract}

Background: We characterised the phenotypic consequence of genetic variation at the PCSK9 locus and compared findings with recent trials of pharmacological inhibitors of PCSK9.

Methods: Published and individual participant level data (300,000+ participants) were combined to construct a weighted PCSK9 gene-centric score (GS). Seventeen randomized placebo controlled PCSK9 inhibitor trials were included, providing data on 79,578 participants. Results were scaled to a one mmol/L lower LDL-C concentration.

Results: The PCSK9 GS (comprising 4 SNPs) associations with plasma lipid and apolipoprotein levels were consistent in direction with treatment effects. The GS odds ratio (OR) for myocardial infarction (MI) was 0.53 ( $95 \% \mathrm{Cl} 0.42$; 0.68), compared to a PCSK9 inhibitor effect of 0.90 (95\% Cl 0.86; 0.93). For ischemic stroke ORs were 0.84 ( $95 \% \mathrm{Cl} 0.57$; 1.22) for the $\mathrm{GS}$, compared to $0.85(95 \% \mathrm{Cl} 0.78 ; 0.93)$ in the drug trials. ORs with type 2 diabetes mellitus (T2DM) were 1.29 (95\% Cl 1.11; 1.50) for the GS, as compared to 1.00 (95\% Cl 0.96; 1.04) for incident T2DM in PCSK9 inhibitor trials. No genetic associations were observed for cancer, heart failure, atrial fibrillation, chronic obstructive pulmonary disease, or Alzheimer's disease - outcomes for which large-scale trial data were unavailable.

Conclusions: Genetic variation at the PCSK9 locus recapitulates the effects of therapeutic inhibition of PCSK9 on major blood lipid fractions and MI. While indicating an increased risk of T2DM, no other possible safety concerns were shown; although precision was moderate.

Keywords: Genetic association studies, Mendelian randomisation, LDL-cholesterol, Phenome-wide association scan

\section{Background}

Statins and ezetimibe reduce the risk of major coronary events and ischemic stroke via lowering of low density lipoprotein-cholesterol (LDL-C) [1-3]. Loss-of-function mutations in PCSK9 are associated with lower LDL-C and a reduced risk of coronary heart disease $(\mathrm{CHD})[4,5]$. Antibodies (mAbs) inhibiting PCSK9, reduce LDL-C in patients with hypercholesterolaemia, and received market access in 2015. The FOURIER and ODYSSEY OUTCOMES trials tested the efficacy of PCSK9-inhibition versus placebo on the background of statin treatment and both found that PCSK9 inhibition led to a 15\% relative risk reduction of major vascular events in patients with established CVD and recent acute coronary syndrome over a median follow up of 2.2 to 2.8 years $[6,7]$.

Evidence is limited on the effect of PCSK9 inhibition on clinical outcomes, and on safety outcomes that might only become apparent with prolonged use. Nor is evidence available on the efficacy and safety of PCSK9 inhibitors in subjects other than the high-risk patients studied in trials. Mendelian randomisation for target validation uses naturally-occurring variation in a gene encoding a drug target to identify mechanism-based consequences of pharmacological modification of the same target [8]. Such studies have previously proved useful in predicting success and failure in clinical trials and have assisted in delineating on-target from off-target actions of first-in-class drugs [9-
13]. For example, previous studies showed that variants in $H M G C R$, encoding the target for statins, were associated with lower concentrations of LDL-C and lower risk of coronary heart disease [9] (CHD), while confirming the ontarget nature of the effect of statins on higher body weight and higher risk of type 2 diabetes (T2DM) [9].

We characterised the phenotypic consequences of genetic variation at PCSK9 in a large, general population sample focussing on therapeutically relevant biomarkers, cardiovascular disease (CVD), individual CVD components and non-CVD outcomes such as cancer, Alzheimer's disease, and chronic obstructive pulmonary disease (COPD). Effect estimates from the genetic analysis were compared to those from intervention trials where the outcomes under evaluation overlapped.

\section{Methods}

We summarise methods briefly here as they have been previously described in detail [14].

\section{Genetic variant selection}

SNPs rs11583680 (minor allele frequency $[\mathrm{MAF}]=0.14$ ), rs11591147 (MAF =0.01), rs2479409 $(\mathrm{MAF}=0.36)$ and rs11206510 (MAF $=0.17)$ were selected as genetic instruments at the PCSK9 locus based on the following criteria: (1) an LDL-C association as reported by the Global Lipids Genetics Consortium (GLGC) [15]; (2) low pairwise linkage 
disequilibrium (LD) $\left(r^{2} \leq 0.30\right)$ with other SNPs in the region (based on 1000 Genomes CEU data); and (3) the combined annotation dependent depletion (CADD) score [16] which assesses potential functionality (see Additional file 1: Table S1).

Previously, we explored the between-SNP correlations (see Additional file 2: Figure S1 of Schmidt et al. 2017 [14]), revealing an $\$ \mathrm{r}^{\wedge} 2 \$$ of 0.26 between rs11206510 and rs11583680, confirming all other SNPs were approximately independent $\left(r^{2} \leq 0.07\right)$. Subsequent adjustment for the residual LD (correlation) structure did not impact results (see Appendix Figure 90 of Schmidt et al. 2017 [14]).

\section{Individual participant-level and summary-level data}

Participating studies (Additional file 1: Table S2) provided analyses of individual participant-level data (IPD) based on a common analysis script (available from AFS), submitting summary estimates to the UCL analysis centre. These data were supplemented with public domain data from relevant genetic consortia (Additional file 1: Table S3). Studies contributing summary estimates to genetic consortia were excluded from the IPD component of the analysis to avoid duplication.

Biomarker data were collected on the major routinely measured blood lipids (LDL-C, HDL-C, triglycerides [TG], total cholesterol [TC]); apolipoproteins A1 [ApoA1] and B $[\mathrm{ApoB}]$, and nominal lipoprotein (Lp)(a); systolic (SBP) and diastolic (DBP) blood pressure; inflammation markers Creactive protein (CRP), interleukin-6 (IL-6), and fibrinogen; haemoglobin; glycated haemoglobin $\left(\mathrm{HbA}_{1 \mathrm{c}}\right)$; liver enzymes gamma-glutamyltransferase (GGT), alanine aminotransferase (ALT), aspartate transaminase (AST), and alkaline phosphatase (ALP); serum creatinine, and cognitive function (standardized to mean 0 , and standard deviation 1, see Additional file 1: Table S5).

We focussed on individual clinical endpoints, rather than composites, which have been assessed in outcome trials, as well as disease end-points commonly seen in patients likely to be eligible for PCSK9 inhibitor treatment. Ischemic CVD endpoints studied were myocardial infarction (MI), ischemic stroke, revascularization, and angina. The following non-ischemic CVD events were considered: haemorrhagic stroke, heart failure, and atrial fibrillation. Non-CVD outcome data was collected on common chronic diseases: COPD, any cancer (including those of the breast, prostate, colon and lung), Alzheimer's disease, and T2DM. Study endpoints and biomarker were chosen based on a combination of 1) available sample size, 2) clinical relevance, and 3) evaluation in RCTs of PCSK9 inhibition, we did not a priori hypothesize on the likelihood of PCSK 9 being associated with any of the available phenotypes. Specific cancer sites evaluated here: chronic lymphocytic leukaemia, multiple myeloma, Hodgkin, meningioma, glioma, melanoma, colorectal cancer, prostate cancer, breast cancer, lung adenocarcinoma, and small-cell lung cancer.

Finally, aggregated trial data on the effect of monoclonal PCSK9 (13 alirocumab trials, and 4 evolocumab trials) inhibitors were compared to placebo for MI, revascularization, ischemic or haemorrhagic stroke, cancer, and T2DM abstracted from the Cochrane systematic review $[6,17]$, with the addition of the OUTCOMES alirocumab trial published afterwards [18]. We compared effects on biomarkers and clinical endpoints common to both the genetic analysis and trials.

\section{Statistical analyses}

In all analyses, we assumed an additive allelic effect with genotypes coded as 0,1 and 2 , corresponding to the number of LDL-C lowering alleles; model comparison tests did not show signs of non-additivity [14]. Continuous biomarkers were analysed using linear regression and binary endpoints using logistic regression. Studyspecific associations were pooled for each SNP using the inverse variance weighted method for fixed effect metaanalysis. Study-specific associations were excluded if the SNP was not in Hardy-Weinberg equilibrium (see Additional file 1: Table S4, based on a Holm-Bonferroni alpha criterion), with no variants failing this test. We estimated the effect at the PCSK9 locus by combining all four SNPs in a gene centric score (GS) as the inverse variance weighted effect of the 4 variants, that were subsequently scaled by the inverse variance weighted effect on LDL-C.

Trial data were assembled as per Schmidt et al. 2017 [6]. Briefly, systematic searches were performed using the Cochrane Central Register of Controlled Trials (CENTRAL), MEDLINE, Embase, Web of Science registries, Clinicaltrials.gov and the International Clinical Trials Registry Platform databases. Data from placebo controlled trials were extracted and combined using the inverse variance weighted method for continuous data and a random-intercept logistic regression model for binary data [6].

Results are presented as mean differences (MD) or odds ratios (OR) with 95\% confidence intervals (CI). Analyses were conducted using the statistical programme $\mathrm{R}$ version 3.4.1 [19]. For study specific estimates please contact AFS.

\section{Results}

Participant level data were available from up to 246,355 individuals, and were supplemented by summary effect estimates from data repositories, resulting in a sample size of 320,170 individuals, including 95,865 cases of MI, 16,437 stroke, 11,920 ischemic stroke, 51,623 T2DM, 54, 702 cancer, 25,630 Alzheimer's disease and 12,412 of COPD. 


\section{Lipid and apolipoprotein associations}

As reported previously [14], the four PCSK9 SNPs were associated with lower LDL-C blood concentrations ranging from $-0.02 \mathrm{mmol} / \mathrm{L}(95 \% \mathrm{CI}-0.03$, $0.02)$ per allele for rs 11583680 to $-0.34 \mathrm{mmol} / \mathrm{L}(95 \%$ CI -0.36; - 0.32) for rs11591147 (See Additional file 2: Figure S1). PCSK9 SNPs associated with a lower LDL-C concentration were also associated with lower concentrations of apolipoprotein $\mathrm{B}$ proportionate to the LDL-C association.

Associations of the GS with the other lipids or apolipoproteins, scaled to a $1 \mathrm{mmol} / \mathrm{L}$ lower $\mathrm{LDL}-\mathrm{C}$ were (Table 1): $0.05 \mathrm{mmol} / \mathrm{L}$ (95\% CI 0.02, 0.07) for HDL-C, $0.07 \mathrm{mmol} / \mathrm{L}(95 \% \mathrm{CI}-0.12,-0.01)$ for TG, $-1.06 \mathrm{mmol} / \mathrm{L}$ (95\% CI -1.12, - 1.00) for TC, $-0.20 \mathrm{~g} / \mathrm{L}$ (95\% CI -0.25 , 0.18) for ApoB, $0.02 \mathrm{~g} / \mathrm{L}(95 \%$ CI -0.01, 0.06) for ApoA1, and $-4.12 \mathrm{mg} / \mathrm{dL}(95 \% \mathrm{CI}-8.62,0.38)$ for $\mathrm{Lp}(\mathrm{a})$.

The associations of the PCSK9 GS with blood-based lipid markers were directionally concordant with effects from treatment trials of therapeutic inhibition of PCSK9 (Fig. 1).

Table 1 Biomarker associations of a PCSK9 gene centric score, effect presented as mean difference (MD) with 95\% confidence interval in brackets with the effects scaled to a $1 \mathrm{mmol} / \mathrm{L}$ decrease in LDL-C

\begin{tabular}{lll}
\hline Biomarker & Total sample size & $\mathrm{MD}(95 \% \mathrm{Cl})$ \\
\hline Lipids related biomarkers & & \\
HDL-C in mmol/L & 314,078 & $0.05(0.02 ; 0.07)$ \\
TG in mmol/L & 298,069 & $-0.07(-0.12 ;-0.01)$ \\
TC in mmol/L & 320,170 & $-1.06(-1.12 ;-1.00)$ \\
ApoA1 in $\mathrm{g} / \mathrm{L}$ & 55,477 & $0.02(-0.01 ; 0.06)$ \\
ApoB in $\mathrm{g} / \mathrm{L}$ & 54,643 & $-0.20(-0.25 ;-0.18)$ \\
LP $[$ a] in $\mathrm{mg} / \mathrm{dL}$ & 21,181 & $-4.12(-8.62 ; 0.38)$ \\
Safety related biomarkers & & \\
SBP in mmHG & 182,487 & $0.03(-0.05 ; 0.10)$ \\
DBP in $\mathrm{mmHG}$ & 182,497 & $0.08(0.001 ; 0.15)$ \\
CRP in log (mg/L) & 91,990 & $0.03(-0.07 ; 0.14)$ \\
IL-6 in log (pmol/L) & 22,370 & $-0.08(-0.21 ; 0.04)$ \\
GGT in log (IU/L) & 69,488 & $0.03(-0.04 ; 0.10)$ \\
Fibrinogen in log(g/dL) & 63,288 & $0.02(-0.01 ; 0.04)$ \\
Hemoglobin in g/L & 52,109 & $1.16(-0.38 ; 2.70)$ \\
ALT in log (IU/L) & 83,223 & $0.03(-0.02 ; 0.08)$ \\
AST in log (IU/L) & 49,556 & $0.01(-0.03 ; 0.05)$ \\
ALP in log (IU/L) & 60,222 & $-0.06(-0.09 ;-0.02)$ \\
Creatinine in umol/L & 100,206 & $0.06(-1.43 ; 1.55)$ \\
\hline
\end{tabular}

Nota bene, TG triglycerides, TC Total cholesterol, ApoA1 Apolipoprotein A1, $A p o B$ Apolipoprotein B, LPa Lipoprotein a, SBP Systolic blood pressure, DBP Diastolic blood pressure, CRP C-reactive protein, IL-6 Interleukin-6, GGT Gamma-glutamyltransferase, ALT Alanine transaminase, AST Aspartate transaminase, ALP Alkaline phosphatase

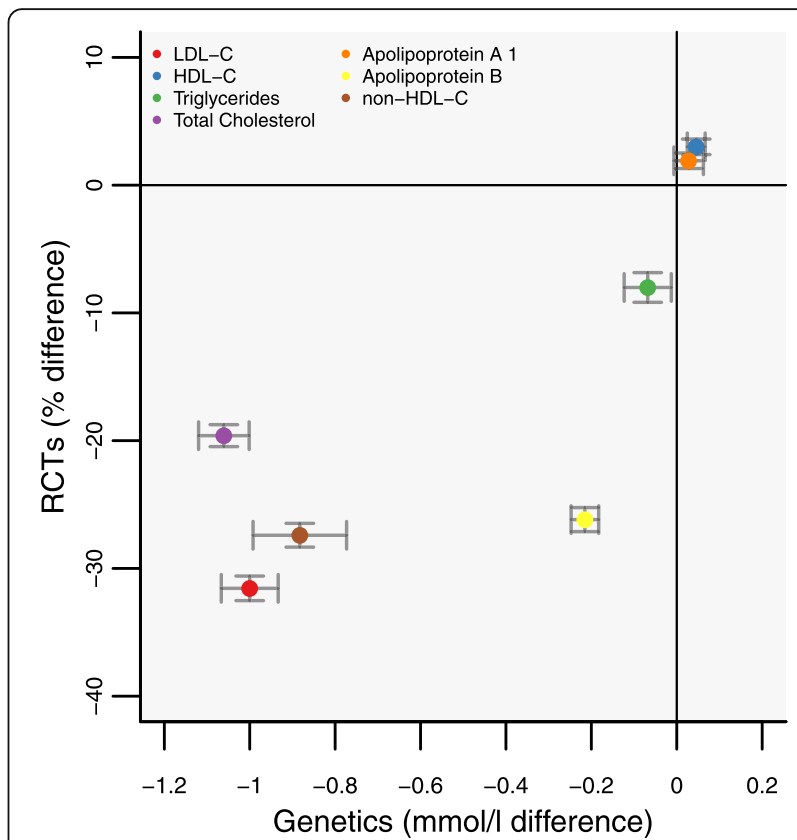

Fig. 1 Lipid and lipoprotein associations of a PCSK9 gene-centric score (GS) compared to placebo-controlled randomized trials of therapeutic inhibition of PCSK9. Footnote: Effect estimates are presented as mean differences, with 95\% confidence interval (Cl). Trial estimates are presented as percentage change from baseline (during 6 months of follow-up), and GS estimates scaled to a $1 \mathrm{mmol} / \mathrm{L}$ lower $\mathrm{LDL}-\mathrm{C}$ (mmol/L). Results are pooled using a fixed effect model. Trial estimates are based on the systematic review by Schmidt et al $2017[6,17]$

\section{Genetic associations with other biochemical and physiological measures}

The GS estimates with SBP and DBP were $0.03 \mathrm{mmHg}$ $(95 \% \mathrm{CI}-0.05,0.10)$ and $0.08 \mathrm{mmHg}(95 \% \mathrm{CI} 0.0001,0.15)$, respectively, per $1 \mathrm{mmol} / \mathrm{L}$ lower LDL-C. The PCSK9 GS was associated with nominally lower ALP (IU/L) -0.06 (95\% CI $-0.09,-0.02)$, but not with other liver enzymes (Table 1).

\section{Genetic associations with ischemic cardiovascular events}

The PCSK9 GS was associated with a lower risk of MI (OR 0.53; 95\% CI 0.42; 0.68; 95,865 cases), which was directionally consistent with results from placebocontrolled PCSK9 inhibition trials: OR 0.90 (95\% CI $0.86,0.93)$, with both estimates scaled to a $1 \mathrm{mmol} / \mathrm{L}$ lower LDL-C (Figs. 2 and 3). The genetic effect estimate for ischemic stroke was OR 0.84 (95\% CI 0.57, $1.22,11,920$ cases), concordant in direction to that of the drugs trials (OR 0.85 95\% CI 0.78; 0.93). Similarly, the PCSK9 GS association with coronary revascularization (OR $0.75 \quad 95 \% \quad$ CI $0.44 ; 1.27$ ) was directionally consistent with the PCSK9 inhibitor trials (OR 0.90; 95\% CI 0.86, 0.93) (Fig. 3). 


\begin{tabular}{|c|c|c|c|}
\hline \multicolumn{3}{|c|}{ Events/Total } & OR, [95\%Cl] \\
\hline \multicolumn{4}{|l|}{ Ischemic CVD } \\
\hline Myocardial infarction & $95865 \quad 246118$ & 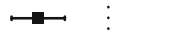 & $0.53[0.42,0.68]$ \\
\hline Revascularization & 1625173434 & & $0.75[0.44,1.27]$ \\
\hline Angina & $2603 \quad 45864$ & & $1.07[0.56,2.06]$ \\
\hline Ischemic stroke & 11920216063 & $\longrightarrow$ & $0.84[0.57,1.22]$ \\
\hline \multicolumn{4}{|l|}{ non-Ischemic CVD } \\
\hline Hemorrhagic stroke & $6267 \quad 204771$ & & $1.29[0.76,2.19]$ \\
\hline Heart failure & $1803 \quad 46330$ & & $1.06[0.48,2.32]$ \\
\hline \multirow[t]{4}{*}{ Atrial fibrillation } & $41485 \quad 311596$ & 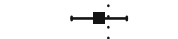 & $0.92[0.72,1.18]$ \\
\hline & & 11 & \\
\hline & & $1 \quad 1.5$ & \\
\hline & Odds Rat & to, scaled per $1 \mathrm{mmol} / \mathrm{L}$ & ase in LDL-C \\
\hline \multicolumn{4}{|c|}{$\begin{array}{l}\text { Fig. } 2 \text { Associations of a PCSK9 gene-centric score with ischemic and } \\
\text { non-ischemic cardiovascular endpoints. Footnote: Effect estimates } \\
\text { are presented as odds ratios (OR), with } 95 \% \text { confidence interval }(C l) \\
\text { scaled to a } 1 \mathrm{mmol} / \mathrm{L} \text { lower } \mathrm{LDL}-\mathrm{C} \text { (mmol/L). Results are pooled } \\
\text { using a fixed effect model. The size of the squares are proportional } \\
\text { to the inverse of the variance }\end{array}$} \\
\hline
\end{tabular}

\section{Genetic associations with non-ischemic cardiovascular} disease

The point estimate for the GS association with hemorrhagic stroke (Fig. 2), OR 1.29 (95\% CI 0.76, 2.19), was discordant to the estimate from PCSK9 inhibitor trials (OR $0.9695 \%$ CI 0.75 ; 1.23) (Fig. 3), although the confidence intervals overlapped. Comparing the association of PCSK9 GS with hemorrhagic and ischemic stroke indicated the GS had a differential effect $(p$-value $=0.02)$. No PCSK9 GS association was observed with atrial fibrillation (OR 0.92 95\% CI $0.72 ; 1.18 ; 41,485$ cases), or heart failure (OR $1.0695 \% \mathrm{CI}$ $0.48 ; 2.32 ; 1803$ cases) (Fig. 2).

\section{Associations with non-cardiovascular disease and related biomarkers}

The PCSK9 GS was not associated with the risk of any cancer (OR 0.97: 95\%CI 0.81; 1.17; 54,702 cases, see Fig. 4), nor with any of 12 specific types of cancer (Additional file 2: Figure S2). We did not observe an association with either Alzheimer's disease or cognitive performance: for Alzheimer's the OR was 0.91 (95\% CI $0.55,1.51)$ and for cognition (per standard deviation) -0.03 (95\% CI $-0.22,0.16)$. As reported before [14] the GS was associated with T2DM (OR 1.29 95\% CI 1.11; 1.50) (Fig. 4), higher body weight $(1.03 \mathrm{~kg}, 95 \%$ CI $0.24,1.82)$, waist to hip ratio $0.006(95 \%$ CI $0.003,0.011)$ and fasting glucose $0.09 \mathrm{mmol} / \mathrm{L}(95 \% \mathrm{CI} 0.02,0.15)$. The OR for COPD was 0.89 (95\% CI 0.67, 1.18).

\section{Discussion}

The genetic findings presented here show that variation in PCSK9 is associated with lower circulating

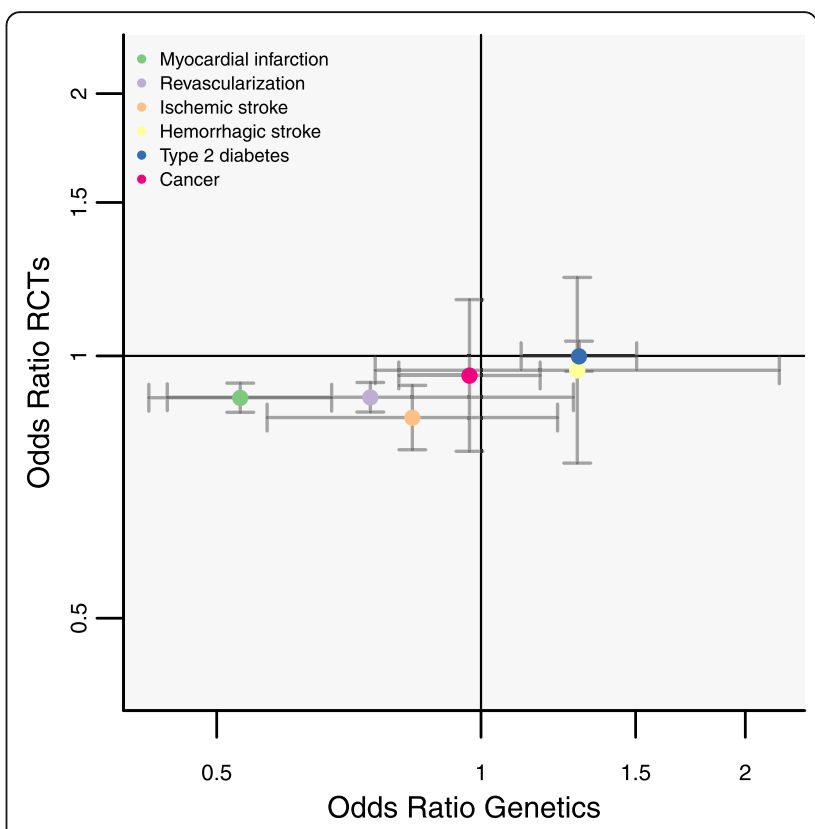

Fig. 3 Clinical endpoint associations of the PCSK9 gene-centric score (GS) as compared to placebo-controlled randomized trials of therapeutic inhibition of PCSK9. Footnote: Effect estimates are presented as odds ratios (OR), with $95 \%$ confidence interval $(\mathrm{Cl})$, for the GS scaled to a $1 \mathrm{mmol} / \mathrm{L}$ lower LDL-C (mmol/L). Results are pooled using a fixed effect model. Trial estimates are based on the systematic review by Schmidt et al 2017 [6], with the estimates on ischemic stroke and revascularization solely based on the FOURIER and ODYSSEY OUTCOMES trials

LDL-C and apoB concentrations, lower risk of MI and, with lesser confidence, the risk of ischemic stroke and coronary revascularization. These effects are consistent in direction to effects observed in PCSK9 inhibitor trial's [20].

A recent systematic review of trial data [21] indicated PCSK9 inhibition was associated with increased fasting glucose $(0.17$ as standardized mean difference [SMD] $95 \%$ CI $0.14 ; 0.19)$ and glycosylated haemoglobin $(0.10$ SMD 95\% CI 0.07, 0.12, 21), although these associations were dependent on the inclusion of the terminated bococizumab trials. Recently we, and others, showed natural genetic variation PCSK9 was associated with elevated fasting glucose and T2DM [14, 22, 23] and that variation at other LDL-C-associated loci also influence risk of T2DM [24, 25]. However, the FOURIER and ODYSSEY OUTCOMES trials, the largest treatment trials of PCSK9 inhibitors to date, did not find an association with risk of incident T2DM, at a median follow up of 2.2 and 2.8 years respectively. It is possible this reflects a genuine discordance between the findings from trials and genetic analyses. Alternatively, the exposure durations in the two largest trials may simply have been too short for subjects to develop T2DM. The risk increasing effect of statins on T2DM was only apparent after conducting a 


Any cancer
Alzheimer

meta-analysis of 13 statin trials in which 4278 T2DM cases were observed during an average follow-up of 4 years [26].

In general, inconsistencies between associations of variants in a gene encoding a drug target and the effects of the corresponding treatment are possible on a number of theoretical grounds. The effects of genetic variation (present from conception) may be mitigated by developmental adaptation or environmental changes. A lack of association of a genetic variant with an outcome therefore does not preclude an effect of a treatment administered in later life, when adaptive responses may no longer be available, or in the presence of a particular environment [27]. We selected a subset of all genetic variants at PCSK9 that capture information on many others and which have some annotated function. However, other approaches to more fully capture the entire gene-centric effect are worthy of future investigation [28].

The association of PCSK9 variants with LDL-C and MI has been reported before [5], and was a motivating factor for the development of PCSK9 inhibiting drugs. Lotta and colleagues [22] reported a similar OR for MI of $0.60(95 \%$ CI $0.48,0.75)$ per $1 \mathrm{mmol} / \mathrm{L}$ decrease in LDL-C using the PCSK9 rs11591147 SNP. Using a seven SNP PCSK9 GS, Ference et al. reported a MI OR of 0.44 (95\% CI $0.31,0.64)$ per $1 \mathrm{mmol} / \mathrm{L}$ decrease in LDL-C [23]. These scaled genetic effects are larger than the treatment effect observed in trials which others have noted previously [29], and ascribed to the lifelong effect of genetic variation versus the short-term effect of drug treatment in later life.
The available trial data showed PCSK9 inhibitors had a similar effect on MI (OR 0.90, 95\% CI 0.86; 0.93) and ischemic stroke (OR 0.85 95\% CI 0.78; 0.93). By contrast, the genetic analysis indicated a directionally concordant, but larger effect on MI (OR 0.53; 95\% CI 0.42; 0.68) than ischemic stroke, (OR 0.84 95\% CI 0.57; 1.22). The genetic analysis was, however, based on only 11,920 stroke cases, about one-fifth of the number of cases available for the genetic analysis of MI and as such confidence interval overlapped. We did observe a differential association between PCKS9 SNPs and ischemic and hemorrhagic stroke (interaction $p$-value $=0.02$ ). Findings from statin trials previously suggested LDL-C lowering through inhibition of HMG-coA reductase is associated with a reduced risk of ischemic but potentially increased risk of hemorrhagic stroke [30-32]. Our findings suggest that a different effect on ischemic and hemorrhagic stroke subtypes may be eventually identified for PCSK9 inhibitors.

Despite previous concerns about a potential effect of this class of drugs on cognition [33], the genetic analysis did not reveal a significant association of the PCSK9 variants with cognitive function or Alzheimer's disease, nor with COPD or cancer, though this does not preclude an effect on such outcomes from drug treatment given in later life. While we explored the associations with any cancer (54,702 events) as well as individual cancer sites (Additional file 2: Figure S2), we did not have data on some clinically relevant cancer types such as endometrial cancer.

This neutral effect on cognition has been recently reported by the EBBINGHAUS study, nested within the FOURIER trial, which reported a non-significant PCSK9 inhibitor effect on multiple measures of cognition confirming (using a non-inferiority design) an absence of effect [33]; it should be noted that similar to the FOURIER, the EBBINGHAUS follow-up time was limited. The absence of an effect on cognition during PCSK9 inhibitor treatment was also observed in the ODYSSEY OUTCOMES trial, which had a median follow-up [7] of 2.8 years.

Drugs (even apparently specific monoclonal antibodies) can exert actions on more than one protein if such targets belong to a family of structurally similar proteins. PCSK9, for example, is one of nine related proprotein convertases [34]. Such 'off-target' actions, whether beneficial or deleterious, would not be shared by variants in the gene encoding the target of interest. In addition, monoclonal antibodies prevent interaction between circulating PCSK9 and LDL-receptor and should not, in theory, influence any intracellular action of the protein [35].

Genetic association studies of the type conducted here tend to examine the risk of a first clinical event, whereas 
clinical trials such as ODYSSEY OUTCOMES focus on patients with established disease, where mechanisms may be modified. Proteins influencing the risk of a first event may also influence the risk of subsequent events, as observed in the case of the target of statin drugs that are effective in both primary and secondary prevention [1]. For this and other reasons [36-38], examination of the effects of PCSK9 variants on the risk of subsequent CHD events in patients with established coronary atherosclerosis is the subject of a separate analysis led by the GENIUS-CHD consortium [38].

\section{Conclusions}

PCSK9 SNPs associated with lower LDL-C predict a substantial reduction in the risk of $\mathrm{MI}$ and concordant associations with a reduction in risk of ischemic stroke, but with a modestly increased risk of T2DM. In this preliminary analysis we did not observe associations with other non-cardiovascular safety outcomes such as cancer, COPD, Alzheimer's disease or atrial fibrillation.

\section{Additional files}

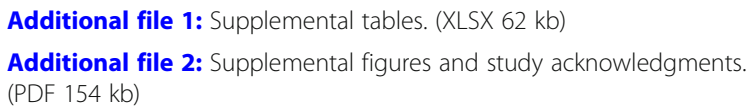

\begin{abstract}
Abbreviations
ALP: Alkaline phosphatase; ALT: Alanine aminotransferase; ApoA1: Apolipoproteins A1; ApoB: Apolipoproteins B; AST: Aspartate transaminase; CADD: Combined annotation dependent depletion; CHD: Coronary heart disease; Cl: Confidence interval; COPD: Chronic obstructive pulmonary disease; CRP: C-reactive protein; CVD: Cardiovascular disease; DBP: Diastolic blood pressure; GGT: Gamma-glutamyltransferase; GLGC: Global lipids genetics Consortium; GS: Gene-centric score; HbA1c: Glycated haemoglobin; IL-6: Interluekin-6; IPD: Individual participantlevel data; LD: Linkage disequilibrium; LDL-C: Low density lipoproteincholesterol; LPa: Lipoprotein a; mAbs: Monoclonal antibodies; MAF: Minor allele frequency; MD: Mean difference; MI: Myocardial infarction; OR: Odds ratio; SBP: Systolic blood pressure; SMD: Standardized mean difference; T2DM: Type 2 diabetes mellitus; TC: Total cholesterol; TG: Triglycerides
\end{abstract}

\section{Acknowledgements}

Not applicable

\begin{abstract}
Authors' contributions
AFS, DIS, MVH, RSP, FWA, JPC, BJK, ADH, DP, NS contributed to the idea and design of the study. AFS, DIS, MVH, designed the analysis scripts shared with individual centres. AFS performed the meta-analysis and had access to all the data. The authors jointly drafted the manuscript, and contributed to subsequent critical revisions. All authors have approved the submitted manuscript, and take responsibility for the integrity and the accuracy of the data and presented results.
\end{abstract}

\section{Funding}

Dr. Schmidt is supported by BHF grant PG/18/5033837. Dr. Preiss is supported by a University of Oxford BHF Centre of Research Excellence Senior Transition Fellowship (RE/13/1/30181). This research has been funded by the British Heart Foundation (SP/13/6/30554, RG/10/12/28456), and the UCL BHF Research Accelerator grant (AA/18/6/24223). The work was also supported by UCL Hospitals NIHR Biomedical Research Centre and by the Rosetrees and
Stoneygate Trusts. This research has been conducted using the UK Biobank Resource under Application Number 12113. The authors are grateful to UK Biobank participants. UK Biobank was established by the Wellcome Trust medical charity, Medical Research Council, Department of Health, Scottish Government, and the Northwest Regional Development Agency. It has also had funding from the Welsh Assembly Government and the British Heart

Foundation. We thank the International Genomics of Alzheimer's Project (IGAP) for providing summary results data for these analyses. The investigators within IGAP contributed to the design and implementation of IGAP and/or provided data but did not participate in analysis or writing of this report. IGAP was made possible by the generous participation of the control subjects, the patients, and their families. The i-Select chips was funded by the French National Foundation on Alzheimer's disease and related disorders. EADI was supported by the LABEX (laboratory of excellence program investment for the future) DISTALZ grant, Inserm, Institut Pasteur de Lille, Université de Lille 2 and the Lille University Hospital. GERAD was supported by the Medical Research Council (Grant $n^{\circ}$ 503480), Alzheimer's Research UK (Grant $n^{\circ}$ 503176), the Wellcome Trust (Grant $n^{\circ}$ 082604/2/07/Z) and German Federal Ministry of Education and Research

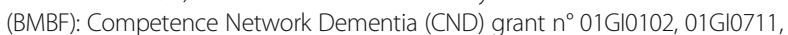
01GI0420. CHARGE was partly supported by the NIH/NIA grant R01 AG033193 and the NIA AG081220 and AGES contract N01-AG-12100, the NHLBI grant R01 HL105756, the Icelandic Heart Association, and the Erasmus Medical Center and Erasmus University. ADGC was supported by the NIH/NIA grants: U01 AG032984, U24 AG021886, U01 AG016976, and the Alzheimer's Association grant ADGC-10-196728. We acknowledge the International Consortium for Blood Pressure Genome-Wide Association Studies (Nature. $2011 \mathrm{Sep}$ 11;478(7367):103-9, Nat Genet. 2011 Sep 11;43(10):1005-11). This work was supported in part by Deutsche Forschungsgemeinschaft (DFG Az Si 552/2), the University of Bonn (BONFOR O-126.0030), and Deutsche Krebshilfe (70/2385/WI2, 70/3163/WI3; PI Prof. J Schramm, Dept. Bloodwise provided funding for the study (LRF05001, LRF06002 and LRF13044) with additional support from Cancer Research UK (C1298/A8362 supported by the Bobby Moore Fund) and the Arbib Fund. Wellcome Trust [064947/Z/01/Z and 081081/Z/06/Z]; from the National Institute on Aging [1R01 AG23522-01]; and the MacArthur Foundation "MacArthur Initiative on Social Upheaval and Health" [71208]. The British Women's Heart and Health Study is supported by the British Heart Foundation (PG/13/66/30442). Data on mortality and cancer events were routinely provided from NHS Digital to the BWHHS under data sharing agreement MR104aRegional Heart Study (Female Cohort). British Women's Heart and Health Study data are available to bona fide researchers for research purposes. Please refer to the BWHHS data sharing policy at www.ucl.ac.uk/british-womens-heart-healthstudy. Hartmut Goldschmidt acknowledges support from the Deutsche Krebshilfe, the Dietmar Hopp Foundation and the German Ministry of Education and Science (BMBF: CLIOMMICS (01ZX1309), Deutsche Krebshilfe, the Dietmar Hopp Foundation and the German Ministry of Education and Science (BMBF: CLIOMMICS (01ZX1309). The Novo Nordisk Foundation Center for Basic Metabolic Research is an independent Research Center at the University of Copenhagen partially funded by an unrestricted donation from the Novo Nordisk Foundation (www.metabol.ku.dk). This study was supported by the Farr Institute of Health Informatics Research, funded by The Medical Research Council (MR/K006584/1), in partnership with Arthritis Research UK, the British Heart Foundation, Cancer Research UK, the Economic and Social Research Council, the Engineering and Physical Sciences Research Council, the National Institute of Health Research, the National Institute for Social Care and Health Research (Welsh Assembly Government), the Chief Scientist Office (Scottish Government Health Directorates) and the Wellcome Trust. Christina M Lill is supported by the Possehl foundation, Renate Maaß Foundation. Mika Kivimaki was supported by MRC (K013351 and R024227) and a Helsinki Institute of Life Science fellowship. Michael Holmes is supported by a British Heart Foundation Intermediate Clinical Research Fellowship (FS/18/23/33512) and the National Institute for Health Research Oxford Biomedical Research Centre. Dr. Patel is supported by a BHF clinical intermediate fellowship (FS/14/76/30933). The funding sources had no role in the design and conduct of the study; collection, management, analysis, and interpretation of the data; preparation, review, or approval of the manuscript; and decision to submit the manuscript for publication.

Lifelines Cohort authors see Additional file 2.

\section{Availability of data and materials}

The datasets used and/or analysed during the current study are available from the corresponding author on reasonable request. 


\section{Ethics approval and consent to participate}

Local ethics committees for studies contributing data to these analyses granted approval for the work.

\section{Consent for publication}

Not applicable

\section{Competing interests}

Dr. Holmes has collaborated with Boehringer Ingelheim in research, and in accordance with the policy of the The Clinical Trial Service Unit and Epidemiological Studies Unit (University of Oxford), did not accept any personal payment. David Preiss consulted for Amgen on a single occasion but, in accordance with the policy of the Clinical Trial Service Unit (University of Oxford), did not accept any personal payment. He is an investigator on a clinical trial of the PCSK9 synthesis inhibitor, inclisiran, funded by a grant to the University of Oxford by the Medicines Company, but he receives no personal fees from this grant. Daniel I Swerdlow is an employee of BenevolentAl Ltd. Aroon Hingorani and Harry Hemingway are National Institute for Health Research Senior Investigators. Naveed Sattar consulted for Amgen and Sanofi related to PCSK9 inhibitors; and was an investigator on clinical trials of PCSK9 inhibition funded by Amgen. Naveed Sattar has also consulted for Boehringer Ingelheim, Janssen, Eli-Lilly and NovoNordisk. Daniel Swerdlow has consulted to Pfizer for work unrelated to this paper. Folkert W. Asselbergs is supported by UCL Hospitals NIHR Biomedical Research Centre Dr. Patel has received honoraria and speaker fees from Sanofi, Amgen and Bayer. Kees Hovingh or his institution (AMC) received honoraria for consultancy, ad boards, and/or conduct of clinical trials from: AMGEN, Aegerion, Pfizer, Astra Zeneca, Sanofi, Regeneron, KOWA, lonis pharmaceuticals and Cerenis. Bertrand Cariou has received research funding from Pfizer and Sanofi, received honoraria from AstraZeneca, Pierre Fabre, Janssen, Eli-Lilly, MSD Merck \& Co., Novo-Nordisk, Sanofi, and Takeda, and has acted as a consultant/advisory panel member for Amgen, Eli Lilly, Novo-Nordisk, Sanofi, and Regeneron. Andrzej Pająk acted as a consultant/advisory pannel member for Amgen. Erik Ingelsson is a scientific advisor for Precision Wellness and Olink Proteomics for work unrelated to this paper. JCH is a scientific advisor to a clinical trial of PCSK9 inhibition. AE Honoraria: Takeda, BMS, Amgen; Consulting: Takeda, BMS, Amgen. SEH acknowledges BHF funding (PG008/08) and support from the UCL BRC. All other authors declare no competing interests.

\section{Author details}

${ }^{1}$ Institute of Cardiovascular Science, University College London, 222 Euston Road, London NW1 2DA, UK. ${ }^{2}$ Department of Cardiology, Division Heart \& Lungs, University Medical Center Utrecht, Utrecht University, Utrecht, the Netherlands. ${ }^{3} \mathrm{UCL}$ 's BHF Research Accelerator Centre, London, UK. ${ }^{4}$ Medical Research Council Population Health Research Unit, Clinical Trial Service Unit \& Epidemiological Studies Unit (CTSU), Nuffield Department of Population Health, University of Oxford, Oxford, UK. ${ }^{5}$ Department of Medicine, Imperial College London, London, UK. ${ }^{6}$ Health Data Research UK, University College London, 222 Euston Road, London NW1 2DA, UK. Institute of Health Informatics, University College London, 222 Euston Road, London NW1 2DA, UK. ${ }^{8}$ The Alan Turing Institute, British Library, 96 Euston Rd, London NW1 2DB, UK. ${ }^{9}$ University College London, Farr Institute of Health Informatics, London, UK. ${ }^{10}$ Clinical Trial Service Unit \& Epidemiological Studies Unit (CTSU), Nuffield Department of Population Health, University of Oxford, Richard Doll Building, Old Road Campus, Roosevelt Drive, Oxford OX3 7LF, UK. ${ }^{11}$ Postgraduate Program in Epidemiology, Federal University of Pelotas, Pelotas, Brazil. ${ }^{12}$ Centre for Population Health Research, Sansom Institute for Health Research, University of South Australia, Adelaide, Australia.

${ }^{13}$ Population, Policy and Practice, UCL GOS Institute of Child Health, London, UK. ${ }^{14}$ South Australian Health and Medical Research Institute, Adelaide, Australia. ${ }^{15}$ Durrer Center for Cardiovascular Research, Netherlands Heart Institute, Utrecht, The Netherlands. ${ }^{16}$ Department of Clinical Epidemiology, Biostatistics And Bioinformatics, Academic Medical Center Amsterdam, Amsterdam, the Netherlands. ${ }^{17}$ Department of vascular medicine, Academic Medical Center Amsterdam, Amsterdam, the Netherlands. ${ }^{18}$ Charité Universitätsmedizin Berlin, corporate member of Freie Universität Berlin, Humboldt-Universität zu Berlin, and Berlin Institute of Health, Lipid Clinic at the Interdisciplinary Metabolism Center, Berlin, Germany. ${ }^{19}$ Charité Universitätsmedizin Berlin, BCRT - Berlin Institute of Health Center for Regenerative Therapies, Berlin, Germany. ${ }^{20}$ Institute of Nutritional Science, University of Potsdam, 14558 Nuthetal, Germany. ${ }^{21}$ Geriatrics Research Group,
Charité - Universitätsmedizin Berlin, 13347 Berlin, Germany. ${ }^{22}$ Department of Nutrition and Gerontology, German Institute of Human Nutrition Potsdam-Rehbruecke, 14558 Nuthetal, Germany. ${ }^{23}$ E.CA Economics GmbH, Berlin, Germany. ${ }^{24}$ Lübeck Interdisciplinary Platform for Genome Analytics (LIGA), Institutes of Neurogenetics \& Cardiogenetics, University of Lübeck, Lübeck, Germany. ${ }^{25}$ Center for Lifespan Changes in Brain and Cognition (LCBC), Dept. Psychology, University of Oslo, Oslo, Norway. ${ }^{26}$ Genetic and Molecular Epidemiology Group, Lübeck Interdisciplinary Platform for Genome Analytics (LIGA), Institutes of Neurogenetics \& Cardiogenetics, University of Lübeck, Lübeck, Germany. ${ }^{27}$ Institute of Human Genetics, Lübeck, Germany. ${ }^{28}$ Ageing Epidemiology Research Unit, School of Public Health, Imperial College, London, UK. ${ }^{29}$ Institute of Genetic Epidemiology, Department of Genetics and Pharmacology, Medical University of Innsbruck, 6020 Innsbruck, Austria. ${ }^{30}$ Department of Neurology, Medical University Innsbruck, Innsbruck, Austria. ${ }^{31}$ Department of Neurology, Inselspital, University Hospital Bern, University of Bern, Bern, Switzerland. ${ }^{32}$ Bradford Institute for Health Research, Bradford Royal Infirmary, Bradford, UK. ${ }^{33}$ Department Primary Care \& Population Health, University College London, London, UK. ${ }^{34}$ Population Health Research Institute, St George's, University of London, London, UK. ${ }^{35}$ Population Health Sciences, University of Bristol, Bristol, UK. ${ }^{36}$ Centre for Population Health Sciences, The Usher Institute, University of Edinburgh, Edinburgh, UK. ${ }^{37}$ Department of Epidemiology and Public Health, UCL Institute of Epidemiology and Health Care, University College London, London, UK. ${ }^{38}$ Department of Medicine, Internal Medicine, Lausanne University Hospital, Lausanne, Switzerland. ${ }^{39}$ Department of Vascular Surgery, Imperial College, London, United Kingdom. ${ }^{40}$ Department of Surgery, Nicosia Medical School, University of Nicosia, Nicosia, Cyprus. ${ }^{41}$ Cyprus International Institute for Environmental and Public Health, Cyprus University of Technology, Limassol, Cyprus. ${ }^{42}$ Julius Center for Health Sciences and Primary Care, University Medical Center Utrecht, Utrecht University, Utrecht, the Netherlands. ${ }^{43}$ Italian Institute for Genomic Medicine (IIGM), Turin, Italy. ${ }^{44}$ Department of Medical Sciences, University of Turin, Turin, Italy. ${ }^{45}$ Unit of Cancer Epidemiology, Città della Salute e della Scienza University-Hospital and Center for Cancer Prevention (CPO), Turin, Italy. ${ }^{46} \mathrm{MRC}$ Epidemiology Unit, Institute of Metabolic Science, University of Cambridge School of Clinical Medicine, Cambridge Biomedical Campus, Addenbrooke's Hospital, Cambridge, UK. ${ }^{47}$ Novosibirsk State Medical University, Novosibirsk Russian Federation. ${ }^{48}$ Institute of Internal and Preventive Medicine, Siberian Branch of the Russian Academy of Medical Sciences, Novosibirsk, Russian Federation. ${ }^{49}$ Department of Epidemiology and Population Studies, Faculty of Health Sciences, Jagiellonian University Medical College, Kraków, Poland. ${ }^{50}$ National Institute of Public Health, Prague, Czech Republic. ${ }^{51}$ Lithuanian University of Health Sciences, Kaunas, Lithuania. ${ }^{52}$ Novo Nordisk Foundation Center for Basic Metabolic Research, Faculty of Health and Medical Sciences, University of Copenhagen, Copenhagen, Denmark. ${ }^{53}$ Department of Clinical Medicine, Faculty of Health and Medical Sciences, University of Copenhagen, Copenhagen, Denmark. ${ }^{54}$ Center for Clinical Research and Prevention, Bispebjerg and Frederiksberg Hospital, Copenhagen, The Capital Region of Denmark, Denmark. ${ }^{55}$ Centre for Cardiovascular Genetics, Department of Medicine, University College London, London, UK. ${ }^{56}$ Center for Human Genetics, Marshfield Clinic Research Institute, Marshfield, USA. ${ }^{57}$ Children's Hospital of Philadelphia, Philadelphia, USA. ${ }^{58}$ University of Minnesota, Minneapolis, USA. ${ }^{59}$ Geisinger, Danville, USA. ${ }^{60}$ Kaiser Permanente Washington Health Research Institute, Seattle, WA, USA. ${ }^{61}$ Department of Biomedical Informatics and Medical Education University of Washington Seattle, Seattle, WA, USA. ${ }^{62}$ Mayo Clinic, Rochester, USA. ${ }^{63}$ Department of Medicine, Department of Pharmacology, Department of Biomedical Informatics, Vanderbilt University School of Medicine, Nashville, TN, USA. ${ }^{64}$ Vanderbilt University, Nashville, USA. ${ }^{65} \mathrm{WH}$, Seattle, USA. ${ }^{66}$ University of Newcastle, Newcastle, NSW, Australia. ${ }^{67}$ Public Health Program, Hunter Medical Research Institute, Newcastle, NSW, Australia. ${ }^{68}$ Hunter New England Local Health District, Newcastle, NSW, Australia. ${ }^{69}$ Population Health Research Institute, Hamilton, Ontario, Canada. ${ }^{70}$ Department of Genetics, University of Groningen, University Medical Center Groningen, Groningen, The Netherlands. ${ }^{71}$ Department of Cardiology, University of Groningen, University Medical Center Groningen, Groningen, The Netherlands. ${ }^{72}$ Department of Epidemiology, University of Groningen, University Medical Center Groningen, Groningen, Netherlands. ${ }^{73}$ Department of Clinical Epidemiology, Leiden University Medical Center, Leiden, The Netherlands. ${ }^{74}$ Department of Medical Sciences, Molecular Epidemiology, Uppsala University, Uppsala, Sweden. ${ }^{75}$ Department of Medicine, Division of Cardiovascular Medicine, Stanford University School of Medicine, Stanford, CA 94305, USA. ${ }^{76}$ Department of Medical Sciences, Molecular 
Epidemiology and Science for Life Laboratory, Uppsala University, Uppsala, Sweden. ${ }^{77}$ Department of Epidemiology, Erasmus University Medical Center, Rotterdam, the Netherlands. ${ }^{78}$ Department of Epidemiology and Biostatistics, Imperial College London, London, UK. ${ }^{79}$ Institute for Community Medicine, University Medicine Greifswald, Greifswald, Germany. ${ }^{80}$ DZHK (German Centre for Cardiovascular Research), partner site Greifswald, Greifswald, Germany. ${ }^{81}$ Chair of Epidemiology, Ludwig-Maximilians-Universität München, UNIKA-T Augsburg, Augsburg, Germany. ${ }^{82}$ Department of Internal Medicine B, University Medicine Greifswald, Greifswald, Germany. ${ }^{83}$ Department of Internal Medicine A, University Medicine Greifswald, Greifswald, Germany. ${ }^{84}$ Interfaculty Institute of Genetics and Functional Genomics, University Medicine Greifswald, Greifswald Germany. ${ }^{85}$ Institute of Health and Wellbeing, University of Glasgow, Glasgow G12 8RZ, Scotland, UK. ${ }^{86}$ Department of Non-Communicable Disease Epidemiology, London School of Hygiene \& Tropical Medicine, London, UK. ${ }^{87}$ The Department of Medical Research, Bærum Hospital, Vestre Viken Hospital Trust, Gjettum, Norway. ${ }^{88}$ Division of Pharmacoepidemiology and Clinical Pharmacology, Utrecht Institute of Pharmaceutical Sciences, Faculty of Science, Utrecht University, Utrecht, The Netherlands. ${ }^{89}$ Department of Respiratory Medicine, Academic Medical Centre, University of Amsterdam, Amsterdam, the Netherlands. ${ }^{90}$ Robertson Centre for Biostatistics, University of Glasgow, Glasgow, UK. ${ }^{91}$ Institute of Genetics and Molecular Medicine, University of Edinburgh, Edinburgh, UK. ${ }^{92}$ Institute of Cardiovascular and Medical Sciences, University of Glasgow, Glasgow G12 8TA, UK. ${ }^{93}$ CNRS UMR 8199, European Genomic Institute for Diabetes (EGID), Institut Pasteur de Lille, University of Lille, 59000 Lille, France. ${ }^{94}$ Department of Genomics of Common Disease, Imperial College London, W12 ONN London, United Kingdom. ${ }^{95}$ INSERM, U-1138, Centre de Recherche des Cordeliers, Paris, France. ${ }^{96}$ UFR de Médecine, Université Paris Diderot, Sorbonne Paris Cité, Paris, France. ${ }^{97}$ Départment de Diabétologie, Endocrinologie et Nutrition, Assistance Publique Hôpitaux de Paris, Hôpital Bicha, Paris, France. ${ }^{98}$ /'institut du Thorax, INSERM, CNRS, UNIV Nantes, CHU Nantes, Nantes, France. ${ }^{99}$ Institute for Social and Economic Research, University of Essex, Essex, UK. ${ }^{100}$ Wellcome Trust Centre for Human Genetics, University of Oxford, Oxford, England. ${ }^{101}$ Boston University School of Medicine, Boston, MA, USA. ${ }^{102}$ Harvard Medical School Center for Cardiovascular Disease Prevention Brigham and Women's Hospital, Boston, USA. ${ }^{103}$ UWash, Seattle, USA. ${ }^{104}$ Penn State, State College, USA. ${ }^{105}$ University of Colorado Denver, Denver, USA. ${ }^{106}$ Division of Genetics and Epidemiology, The Institute of Cancer Research, London, UK. ${ }^{107}$ Div. Molecular Genetic Epidemiology German Cancer Research Center, Im Neuenheimer Feld 580, 69120 Heidelberg, Germany. ${ }^{108}$ Deutsches Krebsforschungszentrum, Heidelberg, Germany. ${ }^{109}$ The Institut du Cerveau et de la Moelle épinière - ICM, Paris, France. ${ }^{110}$ Sorbonne Universités, UPMC Université Paris 06, UMR S 1127, F-75013 Paris, France. ${ }^{111}$ Department of Neurosurgery, Bethel Clinic, Kantensiek 11, 33617 Bielefeld, Germany. ${ }^{112}$ Division of Hematology-Oncology, Department of Pediatrics, Dan L. Duncan Cancer Center, Baylor College of Medicine, Houston, Texas 77030, USA. ${ }^{113}$ Northern Institute for Cancer Research, Newcastle University, Newcastle upon Tyne, UK. ${ }^{114}$ Myeloma Institute for Research and Therapy, University of Arkansas for Medical Sciences, Little Rock, USA. ${ }^{115}$ Department of Hematology, Erasmus MC Cancer Institute, 3075 EA Rotterdam, the Netherlands. ${ }^{116} \mathrm{Hematology}$ and Transfusion Medicine, Department of Laboratory Medicine, BMC B13, SE-221 84 Lund, Sweden. ${ }^{117}$ University Clinic Heidelberg, Internal Medicine $V$ and National Center for Tumor Diseases (NCT), Heidelberg, Germany. ${ }^{118}$ Department of Internal Medicine, University Hospital of Cologne, Cologne, Germany. ${ }^{119}$ Hematology Clinic, Skåne University Hospital, Skåne, Sweden. ${ }^{120}$ Wallenberg Center for Molecular Medicine, Lund University, Lund, Sweden. ${ }^{121}$ The National Institute for Health Research University College London Hospitals Biomedical Research Centre, University College London, 222 Euston Road, London NW1 2DA, UK. ${ }^{122}$ Health Data Research UK and Institute of Health Informatics, University College London, London, United Kingdom. ${ }^{123}$ The Barts Heart Centre, St Bartholomew's Hospital, London, UK. ${ }^{124}$ UPenn, Philadelphia, USA.

${ }^{125}$ Massachusetts Veterans Epidemiology and Research Information Center (MAVERIC) Veterans Affairs Boston Healthcare System, Boston, USA.

Received: 12 April 2019 Accepted: 19 August 2019

Published online: 29 October 2019

\section{References}

1. Collaborators CTT (CTT). Efficacy and safety of cholesterol-lowering treatment: Prospective meta-analysis of data from 90056 participants in 14 randomised trials of statins. Lancet. 2005;366:1267-78.
2. Cannon CP, Blazing MA, Giugliano RP, McCagg A, White JA, Theroux P, et al. Ezetimibe sed to statin therapy after acute coronary syndromes. N Engl J Med. 2015;372(25):2387-97.

3. Bohula EA, Wiviott SD, Giugliano RP, Blazing MA, Park J-G, Murphy SA, et al. Prevention of stroke with the addition of ezetimibe to statin therapy in patients with acute coronary syndrome in IMPROVE-IT. Circulation. 2017. https://doi.org/10.1161/CIRCULATIONAHA.117.029095.

4. Cohen JC, Boerwinkle E, Mosley TH Jr, Hobbs HH. Sequence variations in PCSK9, low LDL, and protection against coronary heart disease. N Engl J Med. 2006;354:1264-72.

5. Benn M, Nordestgaard BG, Grande P, Schnohr P, Tybjaerg-Hansen A. PCSK9 R46L, low-density lipoprotein cholesterol levels, and risk of ischemic heart disease: 3 independent studies and meta-analyses. J Am Coll Cardiol. 2010;55:2833-42.

6. Schmidt AF, Pearce LS, Wilkins JT, Overington JP, Hingorani A, Casas JP. PCSK9 monoclonal antibodies for the primary and secondary prevention of cardiovascular disease. Cochrane Database Syst Rev. 2015;(6):CD011748. https://doi.org/10.1002/14651858.CD011748.

7. Schwartz GG, Steg, Gabriel P, Szarek M, Bhatt DL, Bittner VA, Diaz R, Edelberg JM, Goodman SG, Hanotin C, Harrington RA, Jukema JW, Lecorps G, Mahaffey KW, Moryusef A, Pordy R, Quintero K, Roe MT, Sasiela WJ, Tamby JF, Tricoci P, White HD, Zeiher AM. Alirocumab and Cardiovascular Outcomes after Acute Coronary Syndrome. N Engl J Med. 2018;379:2097107. https://doi.org/10.1056/NEJMoa1801174.

8. Hingorani A, Humphries S. Nature's randomised trials. Lancet. 2005; 366(9501):1906-8.

9. Swerdlow DI, Preiss D, Kuchenbaecker KB, Holmes MV, Engmann JEL, Shah T, et al. HMG-coenzyme A reductase inhibition, type 2 diabetes, and bodyweight: Evidence from genetic analysis and randomised trials. Lancet. 2015;385:351-61.

10. Swerdlow DI, Hingorani AD, Casas JP, Consortium IMR. The interleukin-6 receptor as a target for prevention of coronary heart disease: a mendelian randomisation analysis. Lancet. 2012;379(9822):1214-24.

11. Wensley F, Gao P, Burgess S, Kaptoge S, Di Angelantonio E, Shah T, et al. Association between $\mathrm{C}$ reactive protein and coronary heart disease: mendelian randomisation analysis based on individual participant data. BMJ. 2011;342:d548.

12. Casas JP, Ninio E, Panayiotou A, Palmen J, Cooper JA, Ricketts SL, et al. PLA2G7 genotype, lipoprotein-associated phospholipase A2 activity, and coronary heart disease risk in 10494 cases and 15624 controls of european ancestry. Circulation. 2010;121(21):2284-93.

13. Sofat R, Hingorani AD, Smeeth L, Humphries SE, Talmud PJ, Cooper J, et al. Separating the mechanism-based and off-target actions of cholesteryl ester transfer protein inhibitors with CETP gene polymorphisms. Circulation. 2010; 121:52-62.

14. Schmidt AF, Swerdlow DDI, Holmes MMV, Patel RS, Fairhurst-Hunter Z, Lyall DM, et al. PCSK9 genetic variants and risk of type 2 diabetes: a mendelian randomisation study. Lancet Diabetes Endocrinol. 2016;0(2):735-42.

15. Willer CJ, Sanna S, Jackson AU, Scuteri A, Bonnycastle LL, Clarke R, et al. Newly identified loci that influence lipid concentrations and risk of coronary artery disease. Nat Genet. 2008;40:161-9.

16. Kircher M, Witten DM, Jain P, O'Roak BJ, Cooper GM, Shendure J. A general framework for estimating the relative pathogenicity of human genetic variants. Nat Genet. 2014;46:310-5.

17. Schmidt AF, Pearce LS, Wilkins JT, Overington JP, Hingorani A, Casas JP. PCSK9 monoclonal antibodies for the primary and secondary prevention of cardiovascular disease. Cochrane Database Syst Rev. 2015;(6):CD011748. https://doi.org/10.1002/14651858.CD011748.

18. Tomlinson B, Hu M, Zhang Y, Chan P, Liu ZM. Alirocumab for the treatment of hypercholesterolemia. Expert Opin Biol Ther. 2017;17:633-43.

19. R Core Team. R: A language and environment for statistical computing. Vienna: R Foundation for Statistical Computing; 2017.

20. Dias CS, Shaywitz AJ, Wasserman SM, Smith BP, Gao B, Stolman DS, et al. Effects of AMG 145 on low-density lipoprotein cholesterol levels: results from 2 randomized, double-blind, placebo-controlled, ascending-dose phase 1 studies in healthy volunteers and hypercholesterolemic subjects on statins. J Am Coll Cardiol. 2012;60(19):1888-98.

21. de Carvalho LSF, Campos AM, Sposito AC. Proprotein convertase Subtilisin/ Kexin type 9 (PCSK9) inhibitors and incident type 2 diabetes mellitus: a systematic review and meta-analysis with over 96,000 patient-years. Diabetes Care. 2017. 
22. Lotta LA, Sharp SJ, Burgess S, Perry JRB, Stewart ID, Willems SM, et al. Association between low-density lipoprotein cholesterol-lowering genetic variants and risk of type 2 diabetes. JAMA. 2016;316(13):1383.

23. Ference BA, Robinson JG, Brook RD, Catapano AL, Chapman MJ, Neff DR, et al. Variation in PCSK9 and HMGCR and risk of cardiovascular disease and diabetes. N Engl J Med. 2016;375(22):2144-53.

24. Fall T, Xie W, Poon W, Yaghootkar H, Magi R, Knowles JW, et al. Using genetic variants to assess the relationship between circulating lipids and type 2 diabetes. Diabetes. 2015;64(7):2676-84.

25. White J, Swerdlow DI, Preiss D, Fairhurst-Hunter Z, Keating BJ, Asselbergs FW, et al. Association of Lipid Fractions with Risks for coronary artery disease and diabetes. JAMA Cardiol. 2016;366(6):1108-18.

26. Sattar N, Preiss D, Murray HM, Welsh P, Buckley BM, de Craen AJ, et al. Statins and risk of incident diabetes: a collaborative meta-analysis of randomised statin trials. Lancet. 2010:375:735-42.

27. Smith GD, Ebrahim S. "Mendelian randomization": can genetic epidemiology contribute to understanding environmental determinants of disease? Int J Epidemiol. 2003;32(1):1-22.

28. Burgess S, Zuber V, Valdes-Marquez E, Sun BB, Hopewell JC. Mendelian randomization with fine-mapped genetic data: choosing from large numbers of correlated instrumental variables. Genet Epidemiol. 2017; 41(8):714-25.

29. Burgess S, Butterworth A, Malarstig A, Thompson SG. Use of Mendelian randomisation to assess potential benefit of clinical intervention. BMJ. 2012; 345(nov06 1):e7325.

30. Heart Protection Study Collaborative Group. Effects of cholesterol-lowering with simvastatin on stroke and other major vascular events in 20536 people with cerebrovascular disease or other high-risk conditions. Lancet. 2004;363(9411):757-67.

31. Amarenco P, Bogousslavsky J, Callahan A, Goldstein LB, Hennerici M, Rudolph AE, et al. High-dose atorvastatin after stroke or transient ischemic attack. N Engl J Med. 2006;355(6):549-59.

32. Collins R, Reith C, Emberson J, Armitage J, Baigent C, Blackwell L, et al. Interpretation of the evidence for the effi cacy and safety of statin therapy. Lancet. 2016:388(10059):2532-61.

33. Giugliano RP, Mach F, Zavitz K, Kurtz C, Im K, Kanevsky E, et al. Cognitive function in a randomized trial of Evolocumab. N Engl J Med. 2017;377(7):633-43.

34. Zhang L, Song K, Zhu M, Shi J, Zhang H, Xu L, et al. Proprotein convertase subtilisin/kexin type 9 (PCSK9) in lipid metabolism, atherosclerosis and ischemic stroke. Int J Neurosci. 2016;126(8):675-80.

35. Cariou B, Si-Tayeb K, Le May C. Role of PCSK9 beyond liver involvement Curr Opin Lipidol. 2015;26(3):155-61.

36. Hu Y-JYJ, Schmidt AFAF, Dudbridge F, Holmes MVMV, Brophy JM, Tragante $V$, et al. Impact of selection Bias on estimation of subsequent event risk. Circ Cardiovasc Genet. 2017;10(5):e001616.

37. Zewinger S, Kleber ME, Tragante V, McCubrey RO, Schmidt AF, Direk K, et al. Relations between lipoprotein(a) concentrations, LPA genetic variants, and the risk of mortality in patients with established coronary heart disease: a molecular and genetic association study. Lancet Diabetes Endocrinol. 2017; 5(7):534-43.

38. Patel RS, Asselbergs FW. The GENIUS-CHD consortium. Eur Heart J. 2015; 36(40):2674-6.

\section{Publisher's Note}

Springer Nature remains neutral with regard to jurisdictional claims in published maps and institutional affiliations.

Ready to submit your research? Choose BMC and benefit from:
- fast, convenient online submission
- thorough peer review by experienced researchers in your field
- rapid publication on acceptance
- support for research data, including large and complex data types
- gold Open Access which fosters wider collaboration and increased citations
- maximum visibility for your research: over 100M website views per year
At BMC, research is always in progress.
Learn more biomedcentral.com/submissions

